\title{
ЭПИДЕМИОЛОГИЯ ВСПЫШЕК САЛЬМОНЕЛЛЁЗА В ГРУЗИИ ЗА 2011-2018 гг.
}

\author{
Доктор общественного здравоохранения, Дарсания Т. H. $^{1}$ \\ Доктор медииины, Гаручава Н. $\boldsymbol{Ю .}^{2}$ \\ Доктор медичины, Мурджскнели К. $\Gamma^{3}$ \\ Доктор медицины, Купарадзе М. И. ${ }^{4}$ \\ Грузия, Тбилиси, Тбилисский Государственный Медицинский Университет \\ 1 Департамент питания и возрастной медицины, ассистент-профессор \\ ${ }^{2}$ Департамент эпидемиологии и биостатистики, ассистент-профессор \\ ${ }^{3}$ Департамент здоровья окружающей среды и профессиональной Медицины, ассистент- \\ nрофесcop \\ ${ }^{4}$ Департамент пропедевтики, ассистент-профессор
}

DOI: https://doi.org/10.31435/rsglobal_ws/31012020/6894

\section{ARTICLE INFO}

Received: 24 November 2019

Accepted: 18 January 2020

Published: 31 January 2020

\section{KEYWORDS}

foodborne diseases,

salmonellosis,

risk - factors,

food safety.

\begin{abstract}
The goal of the research was assessment of spread of salmonellosis in Georgia in 2012-2018. For the purpose of the research, we analyzed the data of confirmed and suspected cases of salmonellosis, obtained from the reports of the NCDC and the National Food Agency of Georgia. The results of the research prove that the analysis of outbreaks of given diseases in Georgia is not enough in most cases of outbreaks; risk factors are not always detected. Without completed data, it is impossible to develop and conduct targeted sanitary and hygienic measures. Our study also confirms that for the population it is necessary to create a guide to hygiene measures when buying, storing, preparing, processing food, since the consumers responsible for safety of food together with their producers at home.
\end{abstract}

Citation: Дарсания Т. Н., Гаручава Н. Ю., Мурджикнели К. Г., Купарадзе М. И. (2020) Еpidemiologiya Vspyshek Sal'monellyoza v Gruzii za 2011-2018 gg. World Science. 1(53), Vol.1. doi: 10.31435/rsglobal_ws/31012020/6894

Copyright: (C) 2020 Дарсания Т. Н., Гаручава Н. Ю., Мурджикнели К. Г., Купарадзе М. И. Тhis is an open-access article distributed under the terms of the Creative Commons Attribution License (CC BY). The use, distribution or reproduction in other forums is permitted, provided the original author(s) or licensor are credited and that the original publication in this journal is cited, in accordance with accepted academic practice. No use, distribution or reproduction is permitted which does not comply with these terms.

Актуальность. Эпидемиологические данные по пищевым отравлениям, в том числе сальмонеллёза, часто минимально собираются в развивающихся странах. Даже самые явные вспышки, связанные с пищей, обычно не расследуются должным образом, не регистрируются или остаются незамеченными [6]. Усиление надзора и контроля за пищевыми отравлениями и повышение своевременности выявления вспышек были определены в качестве приоритетов общественного здравоохранения нашим правительством, когда создавала службу по надзору за безопасностью продовольствия.

Безопасность пищевых продуктов является одной из наиболее важных проблем человека и тесно связана со здоровьем, экономическим развитием и социальной стабильностью [2,9]. В последние годы во всём мире произошло множество вспышек заболевания. Несмотря на значительные изменения в области управления производством и безопасности пищевых продуктов, развитые страны по-прежнему сталкиваются с многочисленными и критическими проблемами безопасности пищевых продуктов [3]. На самом деле, несмотря на недавнее введение тщательного законодательства и совершенствование методов производства и хранения пищевых продуктов, болезни пищевого происхождения представляют собой серьёзную проблему для экономических последствий общественного здравоохранения (3). Начиная с 1980 г. ВОЗ программа 
продовольственного надзора по борьбе с болезнями пищевого происхождения работает с FAO (TiradoandSchmidt 2001) [3], чтобы постоянно обновлять информацию о пищевых эпидемиях в европейских странах и предоставлять существенную поддержку национальным органам власти в улучшении, предотвращении и контроле болезней пищевого происхождения, но о последних вспышках сальмонеллёза было сообщено из развитых стран (Соединённые Штаты Америки, Англия, Болгария) [10]. Отсутствие сообщений о вспышках сальмонеллёза в развивающихся странах, скорее всего связано с плохим выявлением и регистрацией этого заболевания в этих странах. По оценкам, в Грузии 95\% вспышек пищевого отравления были вызваны микроорганизмами в 2012-2018гг, в том числе и сальмонеллами [8], поэтому очень важно быть ознакомленным с микробиологическим загрязнением пищевых продуктов и контролировать факторы риска для обеспечения их безопасности.

Дополняемые базы данных из информационных систем здравоохранения являются недорогими технологическими инструментами, которые позволяют получать большой объём информации о состояния здоровья населения. Эти инструменты важны, поскольку они могут использоваться для планирования, организации, работы и оценки действий, служб, программ и политики правительства. Однако для эффективного использования информации важно обеспечить достоверность и надёжность данных. Качество данных необходимо для точной оценки состояния здоровья населения [1]. Системы надзора должны быть эффективными, гибкими и расширяющимися. Однако многие современные системы работают медленно и неэффективно. Они используют устаревшие технологии, которые больше не отвечают потребностям пользователей в управлении, анализе, визуализации и распространении данных [5]. Управление данными и их анализ должны разрабатываться и выполняться с особой тщательностью во избежание разрушительных последствий для общественного здравоохранения или пищевой промышленности [4].

Цель исследования. Изучить и дать оценку распространению сальмонеллёза в Грузии с 2011 по 2018 гг.

Методы и материалы исследования. Для исследования мы проанализировали данные подтверждённых и подозреваемых вспышек сальмонеллёза с 2011 по 2018 год, полученные из отчётов Национального центра контроля заболеваний и общественного здравоохранения Грузии (далее NCDC) и Национального агентства продовольствия (далее NFA) [3], при Министерстве сельского хозяйства Грузии. NCDC собирает отчёты о вспышках, эпидемиологических особенностях, о путях и факторах передачи этих заболеваний, предоставленные государственными и частными институциями здравоохранения. Способствующие факторы определяются как аспекты процесса приготовления и обработки пищевых продуктов, которые могли бы привести к загрязнению патогенным агентом. NFA проводит мониторинг, плановый и внеплановый надзор за качеством пищевых продуктов, также анализ рисков. Мы сравнили данные NFA с данными NCDC. Мы рассчитали одномерные частоты характеристик вспышки, особенности приготовления и потребления пищи, предполагаемый пищевой продукт, метод приготовления, показатели частоты вспышек, число заболеваний, сроки вспышек, распределение по полу и возрасту. Название «виновных» продуктов приводим, как оно было указано в отчёте о вспышке.

Результаты исследования. Сальмонеллёз в Грузии, как и в других странах мира, распространен умеренно. Если оценить распространённость заболевания по регионам Грузии, выявятся повышенные случаи в различных регионах страны, в разные годы, что конечно же связано с вспышками в соответствующих регионах. Каждый год встречаются случаи в Тбилиси, в Квемо Картли, в Аджарии, что связано с особенностями этих регионов.

Картина №1, показывает распределение сальмонеллёза в Грузии в 2011-2018 годах в двух категориях. Инцидентность заболевания характеризуется цикличностью, а в возрастной группе <15 лет инцидентность всегда превышает тот же показатель во всех возрастных группах.

Распределение случаев по месяцам не выражает сезонность этого заболевания. Особенный рост наблюдается в марте и в апреле, что связано со вспышками сальмонеллёза в эти месяцы.

Анализ каждого года показывает следующее: в 2011 году NCDC зарегистрировало 19575 случаев кишечных инфекций, в том числе 118 случаев сальмонеллёза (инцидентность 2,6 на 100.000 населения), встречались как единичные случаи, так и групповые вспышки сальмонеллёза. Данные о причинах сальмонеллёза отсутствуют [4], так как NFA начал 
существование в январе 2011 года и не предоставил данные о безопасности пищевых продуктов (3). Из взятых в этом году 516 образцов пищевых продуктов, 114 не соответствовали установленным показателям качества.

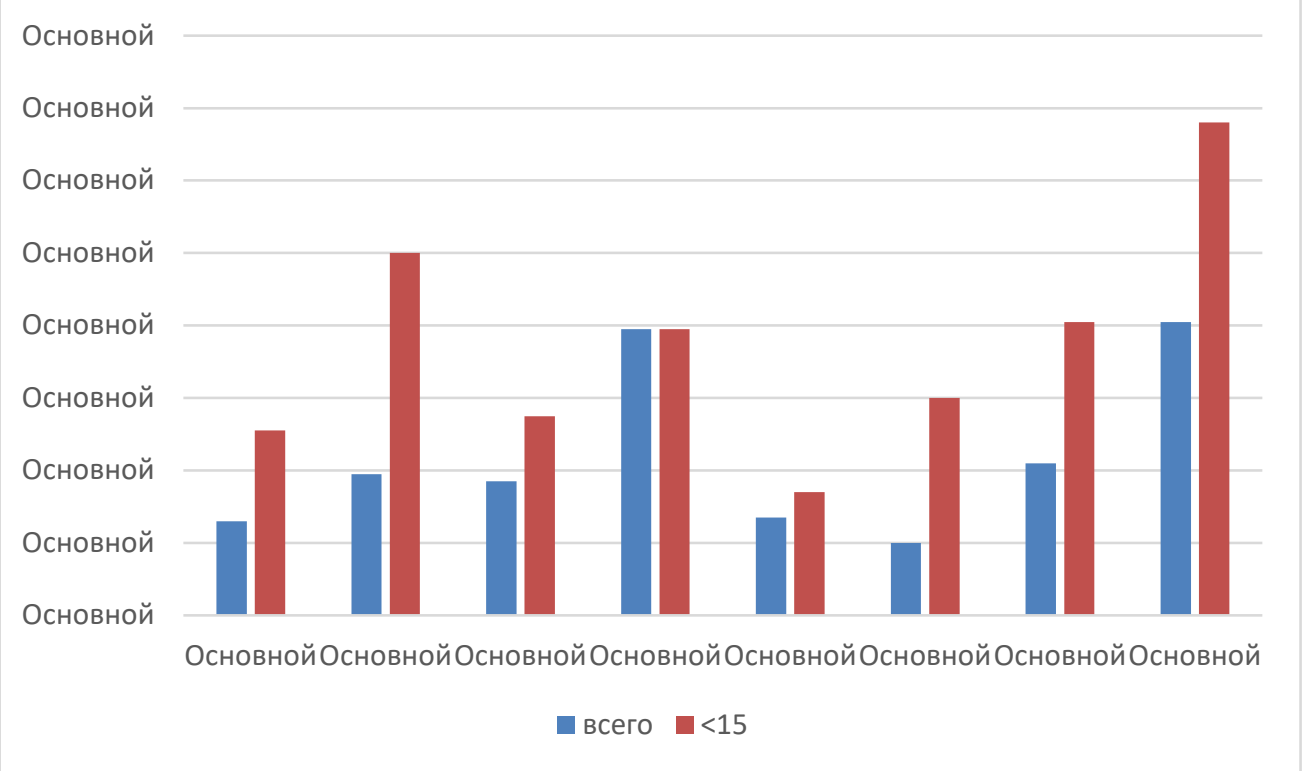

Рис. 1. Распространенность сальмонеллёза в Грузии по возрастным группам в 2011-2018 г2

В 2012 году отмечается рост инцидентности сальмонеллёза в 1,5 раза и составляет 3,9 на 100.000 населения. В этом году возрос показатель среди детей в возрасте младше 15 лет в два раза, что, по нашему мнению, связано с групповой вспышкой в детском саду (фактор передачи не был выявлен). В то время, как NFA не сообщает об этой вспышке, не выявляет загрязнённость пищевых продуктов сальмонеллами и имеются данные только о загрязнении пищевых продуктов условнопатогенными бактериями (коли-бактерии, протеус, сульфитредуцирующие клостридии), в других регионах Грузии также зарегистрированы групповые вспышки сальмонеллёза, предположительным фактором передачи которого был невымытый огурец и торт «птичье молоко». Высокие показатели были выявлены также в возрастной группе 30-59 лет.

В 2013 году растёт число кишечных инфекций за счёт единичных случаев. 3 вспышки сальмонеллёза были зафиксированы за этот год. В первом случае факторами вспышки оказались грибы и «пхали» (варёные огородные овощи с грецкими орехами), в обоих блюдах приправой служила недостаточно вымытая свежая зелень. Во втором случае - мясные блинчики, в третьем - овечий сыр. В этом году почти в два раза снизилась инцидентность этого заболевания среди детей младше 15 лет, что также связано с характером вспышки. По данным NFA 2013 года следует, что из взятых 653 образцов продуктов питания 175 не соответствовали установленным показателям качества. Выявлено большое несоответствие данных двух организаций, которые должны сотрудничать в этом направлении.

В 2014 году, как и в прошедших годах, растут случаи кишечных инфекций, которые, по нашему мнению, вызваны несколькими вспышками сальмонеллёза, показатель которого в два раза превышает показатели 2013 года $(7,9)$. Для выявления фактора передачи инфекции проведено исследование «случай-контроль», конкретный продукт не был выявлен, подозрительными оказались мясные блюда - лабораторное исследование подозрительных продуктов, как и в других случаях прошлых лет, не проводилось. В г. Тбилиси вспышка была связана с употреблением торта, в одном случае, в другом - с молодым сыром. В регионах Грузии случаи сальмонеллёза были связаны с употреблением шаурмы из курицы и жареной рыбой. По данным NFA, из взятых 4190 образцов нарушения зафиксированы в 1059 образцах. Из 501 образца, взятого для исследования сальмонеллёзной загрязнённости, только в 3-х образцах были обнаружены сальмонеллы: шаурма, филе курицы, мясные полуфабрикаты. Листерии были обнаружены в 3-х образцах (готовые блюда) из 201 образца. Сальмонеллёз был распространён в возрастных группах: 30-59, 20-29, 46\% и 23\% соответственно. 
В 2015 году, заболеваемость сальмонеллёза уменьшилась в три раза и составила 2.7, но, как и в предыдущих годах, большинство кишечных инфекций неустановленной этиологии. В детском контингенте распространение составило 7,9 на 100.000 населения, что немного больше, чем в 2014 году.

В 2016 году зарегистрирована одна семейная вспышка сальмонеллёза. Фактор передачи инфекции и в этом случае не обнаружен, но подозрительным продуктом оказалась булочка с кремом. По данным NFA, из 3244 взятых образцов на 6,5\% снизилось число нарушений.

В 2017 году инцидентность болезни в два раза выше (4.2 на 100.000 населения). А среди детей повышение не так высоко (8,1 на 100.000 населения). Случаи сальмонеллёза были зарегистрированы в Тбилиси (80), в Имерети (4), в Кахетии (2), в Квемо Картли (1). В течение отчетного периода в Тбилиси была одна вспышка сальмонеллёза: в марте было зарегистрировано 52 случая заболевания (члены одного ритуального стола). Все заболевшие были старше 15 лет. Результаты лабораторного исследования доказали наличие Salmonella typhimurium в образцах 4 пациентов. Согласно эпидемиологическим исследованиям, высокий фактор шанса был получен в отношении куриного салата (OR = 4,5; CL 1,5-14,1) и шпината (OR = 3,3; CL 1,1-9,7).

В 2018 году инцидентность сальмонеллёза достигла максимального уровня в изучаемый период времени и соответственно составляет 8.1 на 100.000 населения во всех возрастных группах и 13.6 в возрастной группе $<15$ лет.

Обсуждение. Факт, что в большей степени сальмонеллёз встречается в Тбилиси, в Квемо Картли и в Аджарии, можно объяснить следующим образом: Тбилиси является столицей Грузии, где проживают люди из разных регионов Грузии, с различными традициями, приезжают также много туристов и, следовательно, объектов питания, которые способствуют распространению болезни, много. Та же ситуация встречается в Аджарии, которая считается курортной зоной, а распространение болезни в Квемо Картли, по нашему мнению, связано с неудовлетворительными санитарными условиями, а также культурными и санитарными особенностями жителей этого региона. Анализ первой картины показывает, что в стране за последние годы динамика сальмонеллёзных инфекций практически нестабильна. Выходя из эпидемиологического надзора, сальмонеллёз регистрируется единичными случаями только после лабораторного исследования. Исходя из этого, случаи заболевания регистрировались только в тех регионах, где функционируют бактериологические лаборатории. Вероятно, что большинство случаев сальмонеллёза регистрируются как диарея вероятного инфекционного происхождения.

Полученные результаты исследования также свидетельствуют, что анализ вспышек сальмонеллёза в Грузии недостаточно объёмен. В большинстве случаях вспышек риск-факторы не выявляются, особенно при семейных вспышках. Подтверждение диагноза сальмонеллёза основывается на клинико-эпидемиологическом анамнезе. Лабораторное исследование выделений пациентов и подозрительных пищевых продуктов не происходит, особенно в единичных и небольших семейных вспышках. Обнаруживаются несоответствия между данными NCDC и NFA: NFA сообщает о высокой бактериальной обсеменённости пищевых продуктов, готовых блюд, детского питания, а NCDC отмечает случаи неустановленной кишечной инфекции. Надо отметить, что большинство случаев сальмонеллёза встречается среди детского и молодого населения. Кроме этого, надо отметить, что большинство вспышек связано с блюдами домашнего приготовления. При вспышках в детском саду не найден фактор передачи инфекции.

Выводы. Случаи зарегистрированных сальмонеллёзов и мониторинг качества пищевых продуктов не выявляют сути проблем их распространения в Грузии. Ведущее место здесь занимает низкий санитарно-гигиенический уровень населения, сотрудников предприятий, несоблюдение элементарных правил личной гигиены. К тому же отсутствуют данные причин микробного загрязнения пищевых продуктов и готовых блюд, полуфабрикатов промышленного производства, не происходит выявление критических точек на производстве, не исследуются продукты питания лабораторно на микробное загрязнение, не происходит расследование цепи пищевого обсеменения и попадания токсинов, исходя из этого, не выявлены риск-факторы микробного обсеменения. Без этих данных невозможно разработать и провести целенаправленные санитарно-гигиенические мероприятия. Наше исследование также подтверждает, что для населения необходимо создать справочник по соблюдению гигиенических мер при покупке, хранении, приготовлении, обработке пищевых продуктов, так как потребитель ответственен вместе с производителем за безопасность пищевых продуктов в домашних условиях. Особенное внимание надо уделить детям. 


\section{ЛИТЕРАТУРА}

1. Alipour J., Ahmadi M. Dimensios and Assessment Methods of data quality in Health Information Systems. Acta Med. Mediterr. 2017; 33:313-320. doi: 10.19193/0393-6384_2017_2_047. [CrossRef] [Google Scholar]

2. Angelillo IF, Foresta MR, Scozzafava C, Pavia M (2001) Consumers and foodborne diseases: knowledge, attitudes and reported behavior in one region of Italy. Int J Food Microbiol 64:161-166.

3. E. Langiano,M. Ferrara,L. Lanni,V.Viscardi,A.M. Abbatecola, E. De Vito, Food safety at home: knowledge and practices of consumers, Z GesundhWiss.2012 Feb; 20(1): 47-57.

4. Ribot E.M., Hise K.B. Future challenges for tracking foodborne diseases: PulseNet, a 20-year-old US surveillance system for foodborne diseases, is expanding both globally and technologically. EMBO Rep. 2016; 17:1499-1505. doi: 10.15252/embr.201643128. [PMC free article] [PubMed] [CrossRef] [Google Scholar]

5. $\quad$ Richards C.L., Iademarco M.F., Atkinson D., Pinner R.W., Yoon P., Mac Kenzie W.R., Lee B., Qualters J.R., Frieden T.R. Advances in Public Health Surveillance and Information Dissemination at the Centers for Disease Control and Prevention. Public Health Rep. 2017; 132:403-410. doi: 10.1177/0033354917709542. [PMC free article] [PubMed] [CrossRef] [Google Scholar]

6. World Health Organization. In: WHO Estimates of the Global Burden of Foodborne Diseases. 1st ed. WHO Library Cataloguing-in-Publication Data, editor. World Health Organization; Geneva, Switzerland: 2015. [Google Scholar]

7. Национальное агентство продовольствия Грузии, nfa.gov.ge.

8. Национальный центр контроля заболеваний и общественного здоровья Грузии, NCDC.ge.

9. http://www.who.int/topics/infectious_diseases/en/

10. https://www.healthmap.org/en/ 